\title{
CONGENITAL DIVERTICULUM OF THE LEFT VENTRICLE
}

BY

\author{
J. B. LOWE, J. C. P. WILliAMS, D. ROBB, AND D. COLE
}

From the Cardiosurgical Unit, Green Lane Hospital, Auckland, New Zealand

Received May 21, 1958

Congenital diverticula of the ventricles are rare and Skapinker (1951) was able to collect only twelve reported cases. In most of these, as in Skapinker's own patient, the diverticulum arose from the left ventricle but in two it communicated with both ventricles. Skapinker resected the diverticulum in his case, an operation that had previously been reported only once (Roessler, 1944). but further cases treated in this way have since been reported by Potts et al. (1953) and Bailey (1955). Other recent reports are by Formijne (1950), Snellen et al. (1952), and Parsons (1957).

Included in the following description of a patient, in whom surgical resection was successfully undertaken, are electrocardiographic and manometric observations not hitherto recorded. The latter are possibly relevant to the mechanism of rupture of such diverticula, an accident known to have been the cause of death in at least three of the published cases.

\section{CASE REPORT}

The patient, an eight-year-old girl, was referred for surgical treatment of a pulsatile epigastric mass which, because of its superficial position, was considered to be a potential threat to her in the event of trauma. The pulsation had been present at birth and was at that time associated with an umbilical hernia. When she was one year old the hernia had been repaired, the surgeon noting what he took to be an anomalous artery passing up from the epigastrium to disappear under the right costal margin. Visible epigastric pulsation had since persisted without change and without related symptoms. In addition cyanotic congenital heart disease with isolated dextrocardia had been recognized in the neonatal period and the patient had had nonprogressive slight cyanosis and impairment of effort tolerance throughout her life. At the age of eight she was able to run no further than thirty yards. She did not squat and there had been no attacks of severe cyanosis or unconsciousness. The family history and history relating to the maternal pregnancy revealed no unusual features.

Examination showed a thin girl, rather small for her age, with slight central cyanosis and finger clubbing. Physical findings relating to the heart argued for pulmonary hypertension rather than pulmonary stenosis as the cause of shunt reversal; and these, together with the radiographic demonstration of isolated dextrocardia with a left-sided aortic arch and pulmonary vascularity verging on the upper limit of normal (Fig. 1) and the electrocardiogram, consistent in this context with right ventricular hypertrophy (Fig. 4), pointed to a clinical diagnosis of the Eisenmenger complex with dextrocardia. This was supported by the results of cardiac catheterization which included a pulmonary arterial pressure of $112 / 64 \mathrm{~mm}$. $\mathrm{Hg}$, reduction of brachial arterial oxygen saturation to 88 per cent, and a rise in oxygen saturation from 65 per cent in the superior vena cava and right atrium to 70 per cent in the right ventricle and pulmonary artery.

In the epigastrium there was a pulsatile structure which extended upwards from just above the umbilicus to pass under the right costal margin a little to the right of the midline (Fig. 2). The epigastric pulse was slightly delayed as compared to the apex beat, but preceded the carotid arterial pulse by a clinically appreciable interval which was shown by graphic recording to be approximately 0.08 of a second. Over it there was a loud to-and-fro murmur and thrill, and firm pressure on the pulsation just below the costal margin caused this to disappear (Fig. 3) although the pulse below was not appreciably altered. Leads taken with the exploring electrode over the pulsation revealed bizarre patterns consistent with the passage of a wave of excitation in a caudal direction after activation of the main ventricular mass and with subsequent repolarization (Fig. 5); and in the routine electrocardiogram (Fig. 4) S-T segments were distorted by these late 

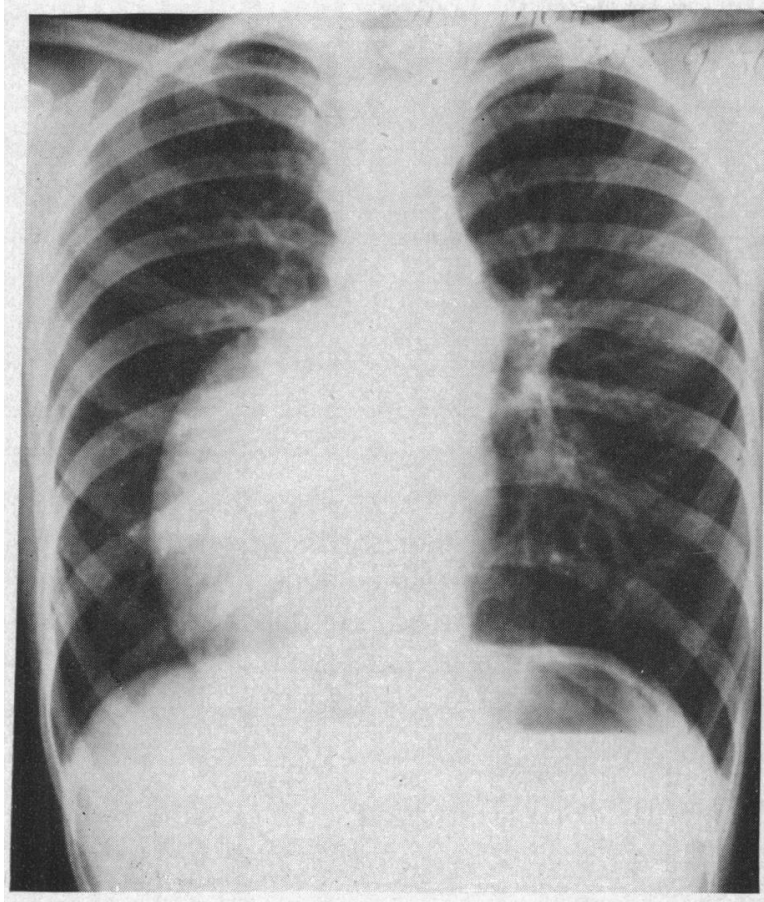

FIG. 1.-Teleradiogram of the heart, showing an isolated dextrocardia and unusual heart contours. Pulmonary vascularity seemed about the upper limit of normal. Fluoroscopy confirmed that the aortic arch was left sided, and excessive hilar pulsation was not seen.

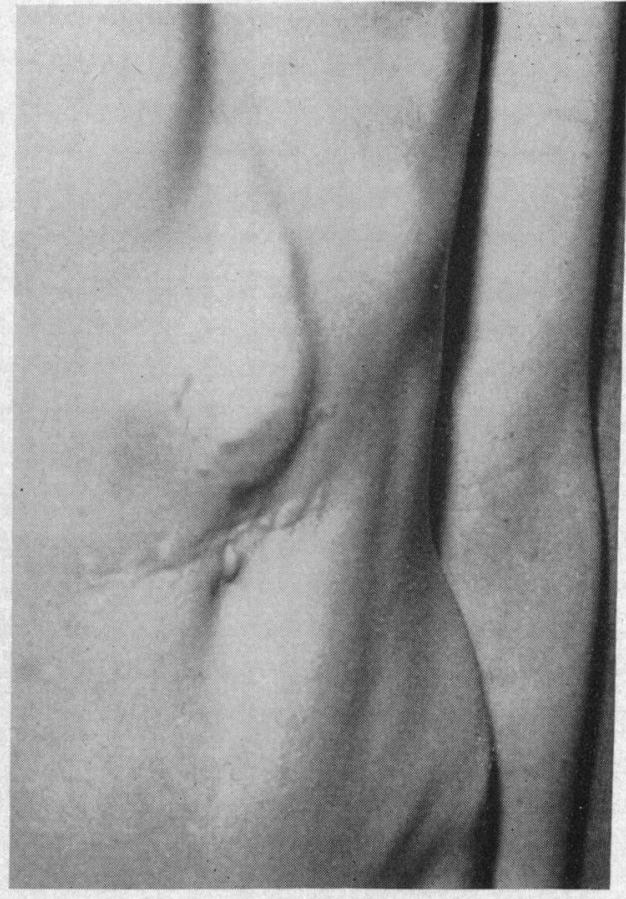

FIG. 2.-Photograph of the abdomen, showing the epigastric mass extending down almost to the umbilicus and the scar of the earlier repair of umbilical hernia.

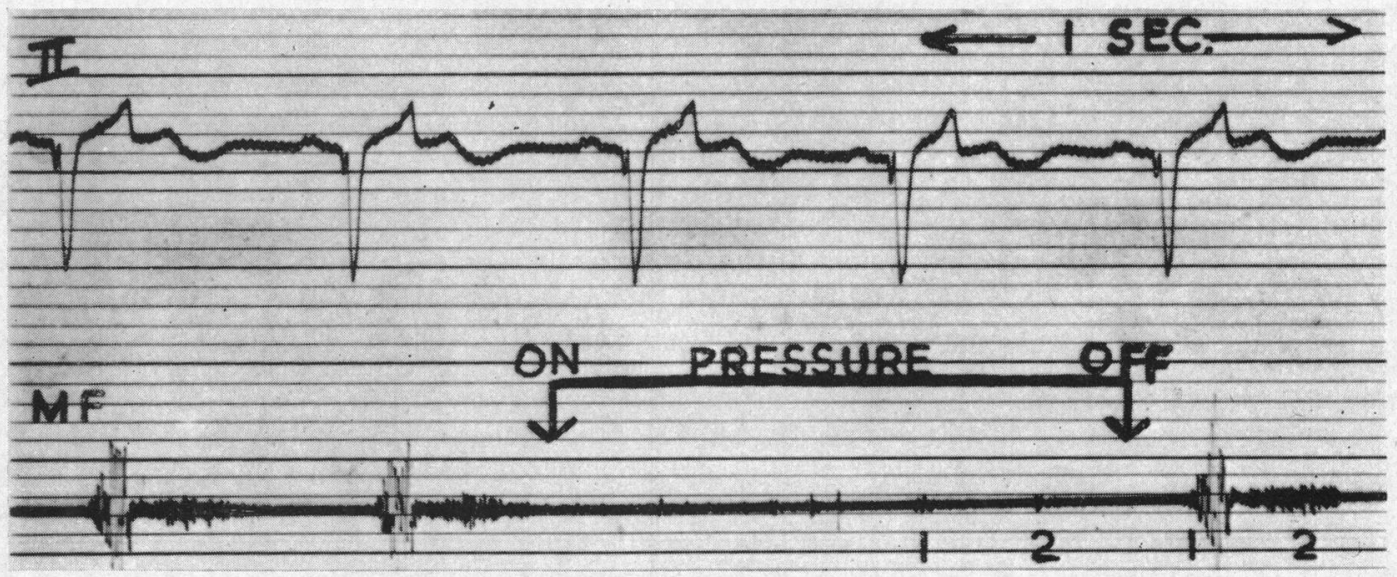

FIG. 3.-Epigastric phonocardiogram, showing abolition of the to-and-fro murmur by pressure over the pulsation at the costal margin. The first component of this murmur begins with the first heart sound and diminishes abruptly before the completion of ventricular systole. The second, in effect a diverticular systolic murmur, begins before the second heart sound and continues into early diastole. 


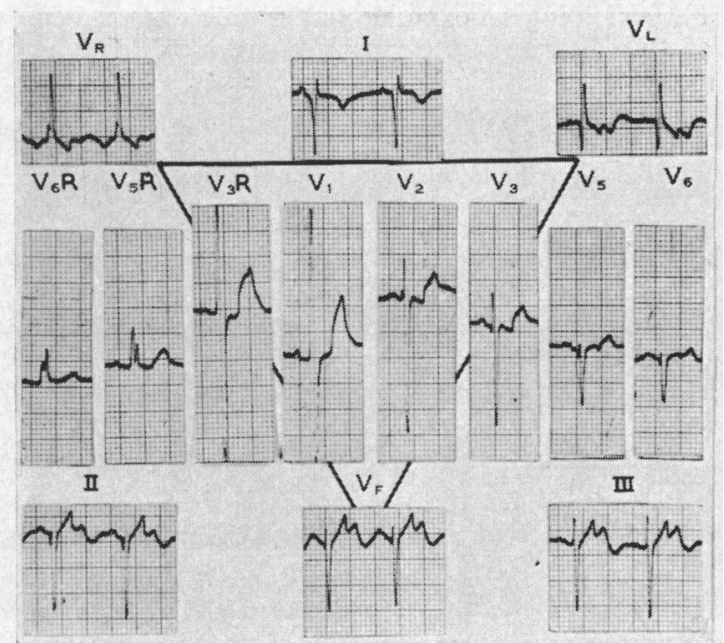

FIG. 4.-The electrocardiogram. The polarity of the $P$ waves argues for a normally sited pacemaker. Ventricular complexes are consistent with moderate hypertrophy of a right ventricle situated behind and to the right of the left ventricle. S-T segments are deformed by deflections, positive in leads II, III, and VF, but negative in VR, VL, and left præcordial leads, which originate in the diverticulum (see text and Fig. 5).

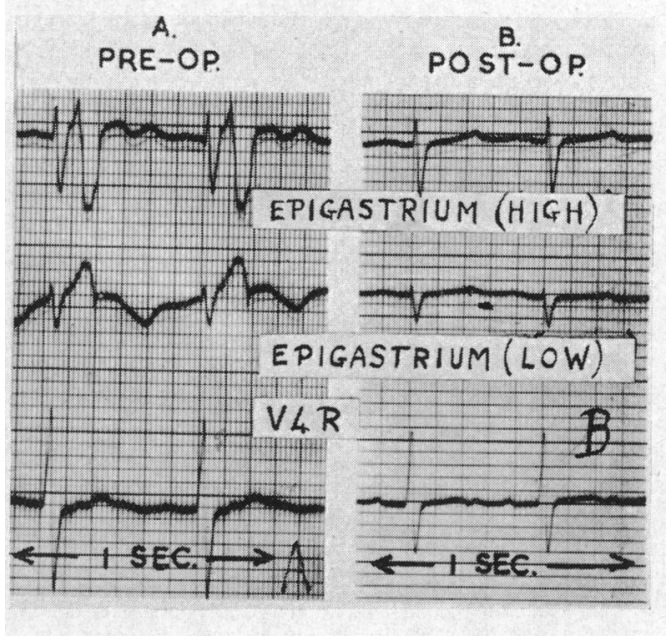

FIG. 5.-V leads from upper and lower epigastrium recorded simultaneously with V4R, (A) before and (B) after surgery. After activation of the main ventricular mass a wave of activation passes down the diverticulum from base to tip and is followed by a repolarization wave of opposite polarity.

potentials. Pressure records obtained by direct puncture of the pulsating structure revealed a ventricular wave form but the systolic pressure of $176 \mathrm{~mm}$. $\mathrm{Hg}$ was approximately double that shown in a femoral arterial tracing taken immediately afterwards, and this relationship to systemic pressure was subsequently confirmed at operation (Fig. 6). The oxygen saturation of blood samples withdrawn simultaneously from these two sites was 88 per cent in each instance. Further confirmation of the diagnosis of diverticulum of the left ventricle was obtained by contrast radiography (Fig. 7).

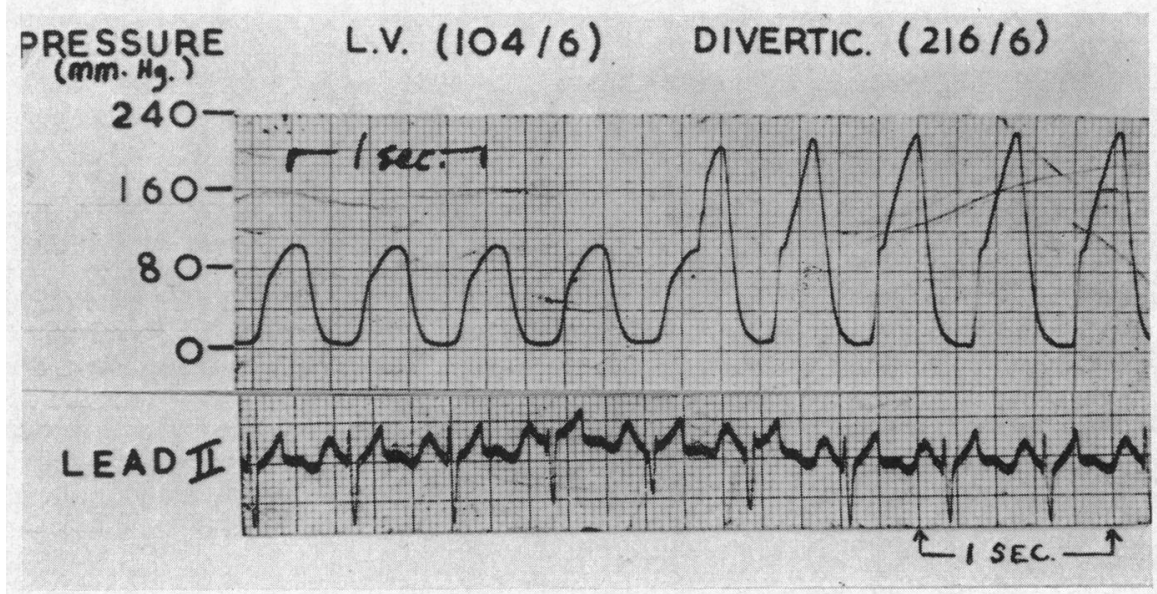

FIG. 6.-Pressures obtained at operation by simultaneous direct puncture of left ventricle and diverticulum and recorded in rapid sequence by means of a three-way connection. Direct tracings at this time showed a pressure of $112 / 70 \mathrm{~mm}$. $\mathrm{Hg}$. in the radial artery.

The Specimen. The diverticulum, which was removed at operation, was a firm muscular tube with a lumen $0.8 \mathrm{~cm}$. in diameter. It was $7.3 \mathrm{~cm}$. in length, with an external diameter of $1 \mathrm{~cm}$. at the open proximal 
end, increasing to $2 \mathrm{~cm}$. towards the distal end (Fig. 8). Histological examination revealed normal cardiac muscle with some irregular strands of fibrous tissue scattered through the muscle bundles and a somewhat thickened fibrous endocardial layer with a normal proportion of elastic tissue. The blood vessels showed no abnormality.

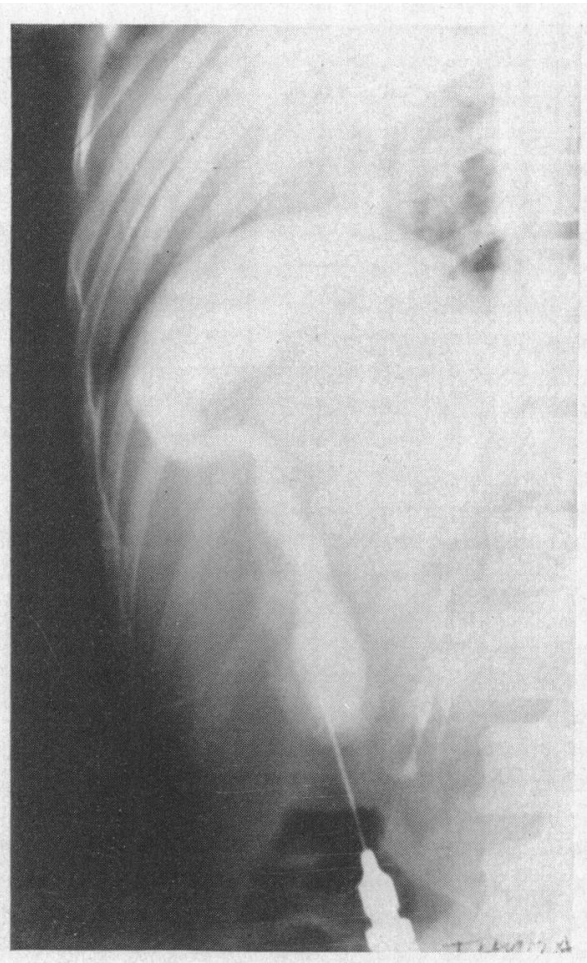

FIG. 7.-Contrast radiogram after injection directly into the diverticulum. The patient has been turned slightly to her right. The course of the diverticulum and its connection with the heart are shown.

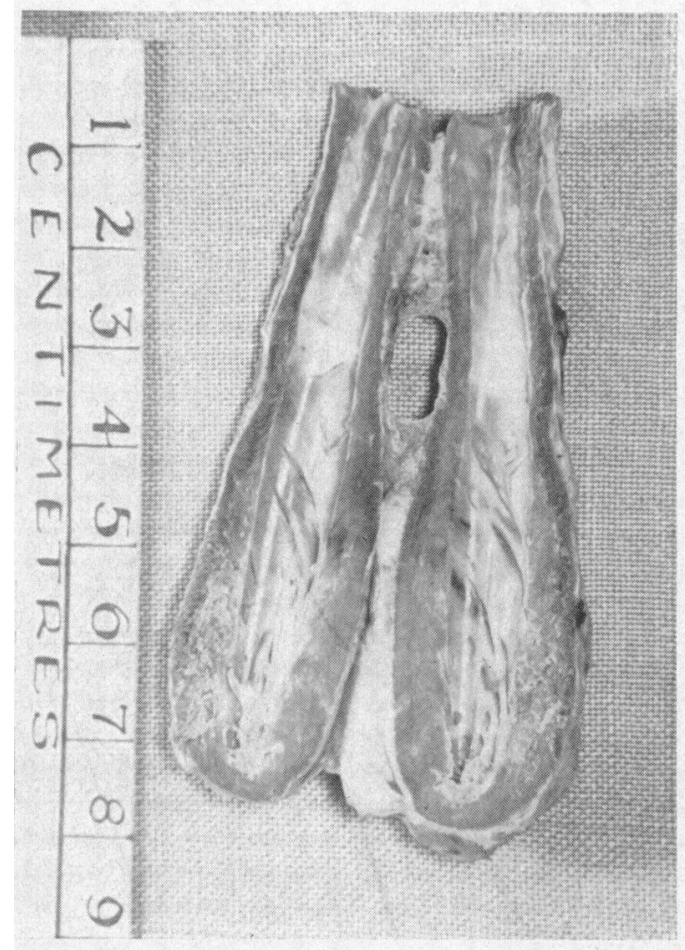

FIG. 8.-The specimen cut longitudinally and opened out. In contrast to the radiographic appearances (Fig. 7) the lumen is almost uniform in calibre.

Operation. In November, 1956, resection of the diverticulum was undertaken by two of us (D. R. and D. C.). Under general anæsthesia, and with a cannula in the right radial artery, a midline epigastric incision was made and carried up in the midline of the sternum and out into the fourth right space. With division of the internal mammary vessels, the right pleura, and the pericardium, a good view was obtained of the left ventricle with the diverticulum passing downwards through a gap bounded by costal margin in front and a rim of diaphragm behind. A tube of pericardium enclosed the diverticulum as it went down, so that the latter lay free in it, except for a mesocardium which attached the ventricle and the medial aspect of the diverticulum to the parietal pericardium. Division of the mesocardium left the tubular diverticulum a free base connecting it to the ventricle which was later clamped across twice with Pott's fine-toothed clamps, and division made between them. Pictures of the beating diverticulum and pressures in it and in the left ventricle were taken. After the first clamp was applied, the diverticulum continued to beat for thirty seconds, then ceased, and later resumed at half the rate of the ventricle. It soon slowed to a third, then a fourth, and then stopped altogether. The removal of the diverticulum was easy, peritoneum being opened in the process. The lower end was blind and lay near the umbilicus. The clamped base was closed by layers of 000 Deknatel as mattress sutures, and was no trouble. An attempt to map out the cardiac anatomy was not persisted with, as the heart was very irritable.

Wound closure, convalescence, and progress were all uneventful and the patient was discharged from hospital some two months later. After operation, neither persistent tachycardia nor cardiac enlargement, noted by Skapinker (1951) and Roessler (1944) in their cases, occurred at any stage. 


\section{Discussion}

The embryological basis for congenital diverticula of the ventricles has been discussed by several authors. Drennan and van der Vijver (1928) and Swyer et al. (1950) postulated that in fœtal life the ventricular wall might give way at a point of weakness, the former authors suggesting that a potentially weak area was present at the apex of the normal heart and the latter that a defect in the muscular attachment to the mitral ring had led to the formation of the diverticulum in their case. On the other hand the frequency with which diverticulum of the ventricle is associated with defects in the pericardium, diaphragm, and abdominal wall was emphasized by Potts et al. (1953): they regarded the diverticulum as only one part of an anomaly that could be attributed to fusion between the part of the primitive cardiac loop that later forms the left ventricle and the closely adjacent ventral portion of the septum transversum prior to the descent of the latter. While this theory appears to offer the more satisfactory explanation for most recorded cases, including our own, it does not necessarily account for all congenital diverticula of the ventricles. In the patient reported by Swyer et al. (1950), where a thin-walled diverticulum not associated with abnormality of the diaphragm or the abdominal wall arose not at the apex but at the base of the left ventricle, the suggestion made by these authors of an underlying structural weakness seems to have much to commend it.

Diagnosis. Provided it is known that this malformation may occur the diagnosis presents no difficulty for the only condition with which it is likely to be confused, an ectopic heart, can be excluded by radiography (Potts et al., 1953). Should the possibility of an anomalous artery be entertained, timing of the pulsation relative to that in the carotid artery should offer a clue to the correct diagnosis, while the direct electrocardiogram alone would probably be diagnostic. Other observations such as pressure recording and contrast radiography can be regarded as being of interest rather than of essential diagnostic importance.

Prognosis and Treatment. Few of the recorded patients, several of whom have had other congenital anomalies, have survived infancy and the only adult so far described was a woman aged thirty, reported by Formijne (1950). In the series of Skapinker (1951) the cause of death was known in only five of ten cases but at least two, one aged six and the other less than two days old, had died from rupture of the diverticulum; and, according to Swyer et al. (1950), rupture of a diverticulum in an eight-year-old child was reported by Von Sydow in 1866. Rupture is therefore not rare and, in the absence of serious associated lesions, it may well be the usual fate of patients with this condition. There can be little doubt that surgical resection is the correct treatment.

Mechanism of Rupture. It has probably not been generally appreciated that systolic pressure in the diverticulum may rise to a level much higher than that in the ventricle. The high pressure found in our patient must have resulted from obstruction to outflow during contraction of the diverticulum but the available data leave the precise nature of the obstruction uncertain. Although the uniform calibre of the lumen excludes organic obstruction, both absolute and relative to the volume of blood contained, in that portion of the diverticulum resected, the possibility of structural narrowing of the lumen as it passed through the ventricular wall remains. On the other hand pressure records confirmed the clinical and cardiographic impression of late contraction of the diverticulum, and revealed that the onset of the systolic pressure rise, about $0.09 \mathrm{sec}$. later than that in the left ventricle, occurred when the ventricle was already in the ejection phase, and therefore at a time when any constriction of its intra-mural portion by the contracting left ventricle would be at or near its maximum. The absence of more than a trivial pressure rise in the diverticulum during isometric ventricular contraction might favour some contribution from organic obstruction, but this evidence is inconclusive for even in this phase the tense left ventricular wall may already have been constricting the diverticulum. Whether or not narrowing of the proximal lumen by muscle contraction in this, the first part of the diverticulum to be activated, contributed to the rise distally is also conjectural, but is made probable by the considerable reduction in calibre of the proximal lumen in some of the films taken in rapid sequence during and after injection of contrast material. Indeed in a thin tubular structure of this type where muscle contraction spreads from open to closed end, a partial systolic obstruction of the open end might possibly lead to a vicious cycle of rise of pressure, muscular hypertrophy, and increasing systolic constriction of the lumen with progressive rise of pressure distally. Certainly this mode of activation of 
the diverticulum offers an attractive explanation for the localization of the greatest muscle mass at the distal closed end of the specimen in the present case, for contraction here would be initiated in the face of a higher pressure than would obtain at the onset of muscular contraction elsewhere in the diverticulum.

In the absence of conclusive evidence as to the relative importance of functional as opposed to organic narrowing of the proximal part of the diverticulum in this case, an estimate of the frequency with which such diverticular pressure will be found in others with this malformation must await further studies. There are, however, some grounds for the belief that it may prove to be the rule rather than the exception and therefore an important factor in the determination of eventual rupture.

\section{SUMMARY}

A patient with cyanotic congenital heart disease of the Eisenmenger type and dextrocardia had an associated diverticulum of the left ventricle. It was successfully removed. Ventricular activation and contraction preceded activation and contraction of the diverticulum, and the systolic pressure in the diverticulum was approximately double that in the left ventricle. The diagnosis and the mechanism of rupture of such diverticula are discussed in the light of these observations.

Our thanks are due to the medical and nursing staff of Green Lane Hospital for co-operation in the investigation and treatment of this patient, to Mr. S. Yarrow for technical assistance, and to the Photographic Unit of the Auckland Hospital Board for the illustrations.

\section{REFERENCES}

Bailey, C. P. (1955). Surgery of the Heart. Henry Kimpton, London.

Drennan, M. R., and van der Vijver, G. T. (1928). J. med. Ass. South Africa, 2, 58.

Formijne, P. (1950). Ned. T. Geneesk, 94, 2704.

Parsons, C. G. (1957). Brit. Heart J., 19, 34.

Potts, W. J., Deboer, A., and Johnson, F. R. (1953). Surgery, 33, 301.

Roessler, W. (1944). Deutsche Ztschr. Chir., 258, 561.

Skapinker, S. (1951). Arch. Surg., 63, 629.

Snellen, H. A., Dankmeijer, J., Bruins, C., and Collister, R. M. (1952) Cardiologia, $21,562$.

Swyer, A. J., Mauss, I. H., and Rosenblatt, P. (1950). Amer. J. Dis. Child., 79, 111.

von Sydow (1866) quoted Swyer et al. (1950). 